\title{
PSEUDO-MULTI-SCALE FUNCTIONS FOR THE STABILIZATION OF CONVECTION-DIFFUSION EQUATIONS ON RECTANGULAR GRIDS
}

\author{
Ali I. Neslitürk* E Onur Baysal \\ Department of Mathematics, Izmir Institute of Technology, 35430, Izmir, Turkey \\ *Address all correspondence to Ali I. Neslitürk E-mail: alinesliturk@iyte.edu.tr
}

We propose a finite element method of Petrov-Galerkin type for a singularly perturbed convection diffusion problem on a discretization consisting of rectangular elements. The method is based on enriching the finite-element space with a combination of multiscale and residual-free bubble functions. These functions require the solution of the original differential problem, which makes the method quite expensive, especially in two dimensions. Therefore, we instead employ their cheap, yet efficient approximations, using only a few nodes in each element. Several numerical tests confirm the good performance of the corresponding numerical method.

KEY WORDS: stabilized FEM, convection-diffusion equation, residual-free bubbles, multiscale functions

\section{INTRODUCTION}

The convection-diffusion equation may exhibit boundary and/or internal layers when the convection process significantly dominates the diffusion. In such cases, the application of the standard Galerkin finite-element method (FEM) with a partition scale that is too big to compute the layers, produces oscillations spreading all over the domain, leading a numerical approximation completely unrelated to the true solution. To accurately resolve the layers, the mesh size must be of the same size as the ratio between the diffusion and the modulus of the convection term. However, that requires an extremely small mesh size, which is not affordable in practical computations in many problems.

Many stabilization techniques were proposed to improve the standard Galerkin FEM in the convection-dominated case, among which, the streamline-upwind Petrov/Galerkin introduced by (Brooks and Hughes, 1982) is one of the most popular. This method corresponds to adding a consistent term providing additional diffusion in the streamline direction to improve the numerical stability of the Galerkin method without compromising accuracy. A wide variety of applications of this method to many interesting problems can be found in the literature (Brezzi and Douglas, 1988; Franca and Frey, 1992; Franca et al., 1992; Franca and Valentin, 2000; Harari and Hughes, 1994; Hughes et al., 1986). However, the amount of additional diffusion should be carefully chosen by the user through a stabilization parameter $\tau$, which is usually seen as a drawback of the method.

One of more recent strategies based on the enrichment of the finite-element spaces, in order to capture the small scales is known as the residual-free bubble (RFB) method introduced by Brezzi and Russo (1994) (see also Brezzi et al. (1998a), Franca et al. (1998), Brezzi et al. (1999), Sangalli (2000)). The RFB strategy maintains the Galerkin method but enhances the polynomial spaces by so-called RFB functions, which satisfy a differential equation inside each element and vanish on its boundary. However, the vanishing boundary condition along interelement boundaries in higher dimensions may lead to inaccuracies in the numerical solution since the approximate solution is still interpolated by polynomials along the element's edges. In fact, the RFB method in advection-dominated problems is less accurate at the outflow boundaries compared to other stabilized methods Franca et al. (2005b). Thus, an improvement in the RFB method is required. Alternatively, a different strategy based on enriching the test space with bubble functions and the trial space with so-called multiscale functions, which satisfy the same differential equation with the RFBs but do not vanish on the element edges, was proposed by Franca et al. (2005a,b,c). However, contrary to the RFBs, the internal 
layers are not well captured by the latter algorithm if the mesh is not aligned with the convection field. Therefore, Franca and his co-workers combined these two approaches and reported that employing the multiscale functions in elements connected to the outflow boundaries and the RFB functions in the rest of the domain increases the accuracy of the numerical approximations considerably Franca et al. (2005c). The common point of these two approaches is that either they employ the exact solutions of the equations defining the enriching functions (those are the bubble functions or the multiscale functions) or their approximations using a very fine mesh inside each element, both of which make the numerical method less practical. Regarding the RFBs, the implementation of the method requires the solution of a local boundary value problem, which may not be easier to solve than the original problem. Therefore, owing to the simplicity of element geometry, researchers have proposed several numerical methods to compute an inexpensive approximate solution to the local problem on a specially chosen subgrid consisting of a few nodes, yet the approximate counterpart of the RFB functions retain the crucial features of the exact RFBs from the convergence point of view Brezzi et al. (2003) and Brezzi et al. (2005).

In this work, we extend the aforementioned idea to the multiscale functions and propose a stable, fully discrete, yet inexpensive numerical method for convection-diffusion problems on rectangular grids. As we simply enrich the test space by bubble functions, to enrich the trial space, we employ the multiscale functions in elements connected to the outflow boundaries and the RFB functions in the rest of the domain. However, the numerical method proposed suggests using suitable approximate counterparts of enriching functions, whose significant feature is that they retain the stabilizing feature of the exact ones. This feature is achieved by using a specially chosen subgrid with a single internal node in the interior of each element in the approximation of the RFBs, which are also known as pseudo-RFBs (PRFBs) (Neslitürk, 2010). Regarding the multiscale functions, they only differ along the element's edges from the bubbles; therefore, we use the same strategy in the element's interior. Along the element's edges, we apply the same method reduced to one dimension, which uses a single additional node per edge to approximate the restriction of the multiscale function on the element's edges. The resulting algorithm numerically performs well and the results are comparable with previous ones found in the literature.

The layout of the paper is as follows. In Section 2 we review enriching the polynomial finite-element spaces by the multiscale functions and recall the corresponding numerical method. In Section 3 we display how to approximate those multiscale functions and their use in the associated numerical method. Numerical results are presented in Section 4.

\section{MULTISCALE METHOD}

Consider the following linear elliptic convection-diffusion problem in a bounded polygonal domain $\Omega \subset \mathbb{R}^{2}$ :

$$
\mathcal{L} u:=-\epsilon \Delta u+\beta . \nabla u=f \text { in } \Omega, \quad u=0 \text { on } \partial \Omega
$$

We assume that diffusion coefficient $\epsilon$ is a positive constant. Let $\mathfrak{T}_{h}$ be a standard partition of $\Omega$ into rectangles $K$ where the intersection of any two elements is a vertex, or an edge, or empty. As usual, the outflow boundary is a subset of $\partial \Omega$ given by

$$
\partial \Omega^{\text {out }}:=\{x \in \partial \Omega \mid \beta . n(x)>0\}
$$

where $n$ is the outward normal to $\partial \Omega$. We denote by $\mathfrak{T}_{h}^{\text {out }}$ the set of elements in $\mathfrak{T}_{h}$ that has at least one boundary intersecting with $\partial \Omega^{\text {out }}$. We introduce the mesh diameter $h=\max _{K \in \mathfrak{T}_{h}}\{\operatorname{diam}(K)\}$ and assume that the source function $f$ and the convection field $\beta=\left(\beta_{1}, \beta_{2}\right)$ are piecewise constants with respect to the decomposition $\mathfrak{T}_{h}$. Without loss of generality we take components of $\beta$ are positive in each element $K$. As usual, we denote the inner product in $L^{2}(\mathfrak{D})$ by $(., .)_{\mathfrak{D}}$, where $\mathfrak{D}$ is an open subset of $\Omega$. To simplify the notation for the case $D=\Omega$, we just write $(.,$.$) .$

The weak formulation of problem (1) reads: Find $u \in H_{0}^{1}(\Omega)$ such that

$$
a(u, v)=(f, v), \quad \forall v \in H_{0}^{1}(\Omega)
$$

where the bilinear form $a: H_{0}^{1}(\Omega) \times H_{0}^{1}(\Omega) \rightarrow \mathbb{R}$ is given by

$$
a(u, v):=\epsilon(\nabla u, \nabla v)+(\beta . \nabla u, v)
$$


Due to the coercivity of the bounded bilinear form $a(.,$.$) over H_{0}^{1}(\Omega)$, the weak problem (2) is well posed by the Lax-Milgram theorem. We choose a finite-dimensional subspace $V^{1}(\Omega)$ of $H_{0}^{1}(\Omega)$ as

$$
V^{1}(\Omega)=\left\{v \in H_{0}^{1}(\Omega):\left.v\right|_{K} \quad \text { is a bilinear polynomial } \forall K \in \mathfrak{T}_{h}\right\}
$$

in order to obtain the standard Galerkin method associated with the weak problem (2), so that it reads: Find $u^{h} \in$ $V^{1}(\Omega)$ such that

$$
a\left(u^{h}, v^{h}\right)=\left(f, v^{h}\right), \quad \forall v^{h} \in V^{1}
$$

It is well known that method (5) yields a poor approximation when $\epsilon \ll|\beta| h$ and produces spurious oscillations polluting all over the domain. Since we are interested in finding a finite-element discretization for Eq. (2) that is both stable and accurate on coarse meshes, we enrich the finite-element spaces in the Petrov-Galerkin framework, so that we redefine the trial and test spaces, respectively, as

$$
\begin{aligned}
U^{h}(\Omega): & =V^{1}(\Omega) \oplus E^{h}(\Omega) \\
W^{h}(\Omega): & =V^{1}(\Omega) \oplus B^{h}(\Omega)
\end{aligned}
$$

where

$$
B^{h}(\Omega):=\left\{v \in H_{0}^{1}(\Omega):\left.v\right|_{K} \in H_{0}^{1}(K), \quad \forall K \in \mathfrak{T}_{h}\right\}
$$

and the multiscale space $E^{h}(\Omega) \subset H_{0}^{1}(\Omega)$ will be defined later. Problem (5) now reads: Find $u^{h} \in U^{h}(\Omega)$ such that

$$
a\left(u^{h}, v^{h}\right)=\left(f, v^{h}\right), \quad \forall v^{h} \in W^{h}(\Omega)
$$

Since typical member $u^{h}$ of $U^{h}(\Omega)$ can be split into a bilinear part $u^{1} \in V^{1}(\Omega)$ and a multiscale part $u^{e} \in E^{h}(\Omega)$, solving (9) is equivalent to finding $u^{h}=u^{1}+u^{e} \in V^{1}(\Omega) \oplus E^{h}(\Omega)$ such that

$$
\begin{array}{ll}
a\left(u^{h}, v^{1}\right)=\left(f, v^{1}\right), & \forall v^{1} \in V^{1}(\Omega) \\
a\left(u^{h}, v^{b}\right)=\left(f, v^{b}\right), & \forall v^{b} \in B^{h}(\Omega) .
\end{array}
$$

We note that the last equation is automatically satisfied if we choose $u^{e}$ such that for all $K \in \mathfrak{T}_{h}$

$$
\mathcal{L} u^{e}=f-\mathcal{L} u^{1} \text { in } K
$$

However, in order to find $u^{e}$ uniquely, we should impose an appropriate boundary condition and that condition is given by the solution of the following differential equation:

$$
\mathcal{L}_{\partial K} u^{e}=f-\mathcal{L}_{\partial K} u^{1} \text { on } \partial K, \quad u^{e}=0 \text { at the vertices of } K
$$

where $\mathcal{L}_{\partial K}$ is a differential operator defined on $\partial K$ and explicitly given by

$$
\mathcal{L}_{\partial K} w:=-\epsilon \frac{\partial^{2} w}{\partial s^{2}}+\mathbf{P}(\beta, s) \frac{\partial w}{\partial s}
$$

Here, $s$ is a variable that parameterizes $\partial K$ by arc length and $\mathbf{P}(\beta, s)$ is the usual projection of the convection field onto $\partial K$. Now we introduce $E^{h}(\Omega)$ with its basis set $\left\{\phi_{i}\right\}_{i \in I_{0}} \cup\left\{\phi_{f}\right\}$, where $I_{0}$ is the set of indexes of internal nodal points with respect to the discretization of $\Omega$ and $\phi_{i}$ and $\phi_{f}$ are enriching basis functions, defined by the following auxiliary problems. Denoting the bilinear basis functions by $\psi_{i}$



and

$$
\left\{\begin{array} { r l } 
{ \mathcal { L } \phi _ { f } = f } & { \text { in } K } \\
{ \phi _ { f } = v _ { f } } & { \text { on } \partial K }
\end{array} \quad \text { where } \quad \left\{\begin{array}{rl}
\mathcal{L}_{\partial K} v_{f}=f & \text { on } \partial K \\
v_{f}=0 & \text { at the vertices of } K
\end{array}\right.\right.
$$


Then, $u^{h} \in U_{h}(\Omega)$ can be represented in terms of enriched basis functions

$$
u^{h}=u^{1}+u^{e}=\sum_{i \in I_{0}} u_{i} \lambda_{i}+\phi_{f}
$$

where $\lambda_{i}:=\phi_{i}+\psi_{i}$. We note that the computation of $\lambda_{i}$ is more practical than the computation of $\phi_{i}$. Therefore, we directly calculate $\lambda_{i}$ and use them in Eq. (10) so that the final form of the discrete formulation of the multiscale method reads: Find $u_{i}$ such that

$$
\sum_{i \in I_{0}} a\left(\lambda_{i}, \psi_{j}\right) u_{i}=\left(f, \psi_{j}\right)-a\left(\phi_{f}, \psi_{j}\right)
$$

Before we solve problem (18), we have to find $\lambda_{i} \in U^{h}(\Omega)$ for all $i \in I_{0}$ and $\phi_{f}$, explicitly. In the literature, this task was accomplished by either exactly solving Eqs. (15) and (16) in strong form or using an expensive numerical method to approximate $\lambda_{i}$ on a fine mesh set inside each element, both of which leads to unpractical implementations. Therefore, we want to compute those basis functions by using a cheap, yet efficient approximation method and use the resulting approximate basis functions in Eq. (18) in place of their exact counterparts. The explicit computation of such approximate basis functions is the subject of the following section.

\section{COMPUTING ENRICHED FUNCTIONS}

In this section we will present how to construct the approximate basis functions, which we compute on a specially chosen subgrid defined in a typical element $K$ (see Fig. 1). Without loss of generality, we may assume that the lowerleft vertex of the element is located at the origin and corresponds to the $i$ th node in the discretization. We only display the computation of $\lambda_{i}$, as the other enriched functions can be found in exactly the similar manner.

Let us rewrite problem (15) in terms of the enriched basis function $\lambda_{i}$

$$
\mathcal{L} \lambda_{i}=0 \text { in } K, \quad \lambda_{i}=\theta_{i} \text { on } \partial K
$$

Here, $\theta_{i}$ is the restriction of $\lambda_{i}$ to $\partial K$; that is

$$
\theta_{i}:=\left.\psi_{i}\right|_{\partial K}+\left.\phi_{i}\right|_{\partial K}
$$

and

$$
\mathcal{L}_{\partial K} \theta_{i}=0 \text { on } \partial K, \quad \theta_{i}=\psi_{i} \text { at the vertices of } K
$$



FIG. 1: A typical element $K$. 
Since $\lambda_{i}$ and its restriction to $\partial K$ satisfy Eqs. (19) and (21), the solution can be written as

$$
\lambda_{i}(x, y)=\lambda_{i}^{x}(x) \lambda_{i}^{y}(y)
$$

where

$$
\lambda_{i}^{x}(x):=\left.\theta_{i}\right|_{z_{1}} \text { and } \lambda_{i}^{y}(y):=\left.\theta_{i}\right|_{z_{4}}
$$

and the single variable functions $\lambda_{i}^{x}$ and $\lambda_{i}^{y}$ satisfy

$$
\begin{gathered}
\left.\mathcal{L}_{\partial K}\right|_{z_{1}} \lambda_{i}^{x}=-\epsilon \frac{d^{2} \lambda_{i}^{x}}{d x^{2}}+\beta_{1} \frac{d \lambda_{i}^{x}}{d x}=0 \text { for } x \in\left(0, h_{x}\right), \quad \lambda_{i}^{x}(0)=1 \text { and } \lambda_{i}^{x}\left(h_{x}\right)=0 \\
\left.\mathcal{L}_{\partial K}\right|_{z_{4}} \lambda_{i}^{y}=-\epsilon \frac{d^{2} \lambda_{i}^{y}}{d y^{2}}+\beta_{2} \frac{d \lambda_{i}^{y}}{d y}=0 \text { for } y \in\left(0, h_{y}\right), \quad \lambda_{i}^{y}(0)=1 \text { and } \lambda_{i}^{y}\left(h_{y}\right)=0
\end{gathered}
$$

However, those functions should be brought to the form of bubble functions before we suggest a suitable subgrid. That is, they should vanish at the boundary of the domain where the equations are posed, without upsetting the nature of the differential operator. To bring that end, let us define two auxiliary functions $\alpha_{i}^{x}$ and $\alpha_{i}^{y}$ by

$$
\begin{aligned}
\alpha_{i}^{x}(x) & :=\lambda_{i}^{x}(x)-\psi_{i}(x, 0) \\
\alpha_{i}^{y}(y) & :=\lambda_{i}^{y}(y)-\psi_{i}(0, y)
\end{aligned}
$$

that, from Eqs. (24)-(27), obviously satisfy

$$
\begin{aligned}
& -\epsilon \frac{d^{2} \alpha_{i}^{x}}{d x^{2}}+\beta_{1} \frac{d \alpha_{i}^{x}}{d x}=\frac{\beta_{1}}{h_{x}} \text { for } x \in\left(0, h_{x}\right), \quad \alpha_{i}^{x}(0)=\alpha_{i}^{x}\left(h_{x}\right)=0 \\
& -\epsilon \frac{d^{2} \alpha_{i}^{y}}{d y^{2}}+\beta_{2} \frac{d \alpha_{i}^{y}}{d y}=\frac{\beta_{2}}{h_{y}} \text { for } y \in\left(0, h_{y}\right), \quad \alpha_{i}^{y}(0)=\alpha_{i}^{y}\left(h_{y}\right)=0
\end{aligned}
$$

Now we can add specially chosen internal nodes into domains $\left(0, h_{x}\right)$ and $\left(0, h_{y}\right)$, on which we approximate $\alpha_{i}^{x}$ and $\alpha_{i}^{y}$, so that the resulting approximations, say $\tilde{\alpha}_{i}^{x}$ and $\tilde{\alpha}_{i}^{y}$, retain the stabilizing features of $\alpha_{i}^{x}$ and $\alpha_{i}^{y}$. The location of the additional node is crucial for the stabilization and its choice depending on different configurations can be found in the literature (Brezzi et al., 2003; Neslitürk, 2006). A straightforward application of the asserted approach to problem (28) results in

$$
\tilde{\alpha}_{i}^{x}(x)=\left\{\begin{array}{ll}
\frac{\beta_{1}\left(h_{x}-P\right) x}{2 h_{x} \epsilon}, & x \leq P \\
\frac{\beta_{1} P\left(h_{x}-x\right)}{2 h_{x} \epsilon}, & x>P
\end{array} \quad \text { where } \quad P= \begin{cases}h_{x}-2 \epsilon / \beta_{1}, & \epsilon \leq \beta_{1} h_{x} / 4 \\
h_{x} / 2, & \text { otherwise. }\end{cases}\right.
$$

where $\tilde{\alpha}_{i}^{y}(y)$ is similarly obtained by replacing $x$ by $y, h_{x}$ by $h_{y}$ and $\beta_{1}$ by $\beta_{2}$ in Eq. (30). Thus, recalling Eqs. (22), (26), and (27), the approximate basis function $\tilde{\lambda}_{i}$ can be written as

$$
\tilde{\lambda}_{i}(x, y)=\left[\tilde{\alpha}_{i}^{x}(x)-\psi_{i}(x, 0)\right]\left[\tilde{\alpha}_{i}^{y}(y)-\psi_{i}(0, y)\right]
$$

A comparison of $\lambda_{i}$ and its approximate counterpart $\tilde{\lambda}_{i}$ is given on a patch of four rectangular elements in Figs. 2-4 for decreasing values of $\epsilon$. It is remarkable that although a few additional nodes are used in each element, the results are very comparable with the exact solution. Therefore, it is quite reasonable to employ the approximate enriched functions $\tilde{\lambda}_{i}$ to solve Eq. (18) in place of $\lambda_{i}$, which we call the pseudo-multi-scale method (PMS). 



FIG. 2: $\lambda_{i}$ (left) and $\tilde{\lambda}_{i}$ (right) for $\epsilon=0.1$.


FIG. 3: $\lambda_{i}$ (left) and $\tilde{\lambda}_{i}$ (right) for $\epsilon=0.01$.


FIG. 4: $\lambda_{i}$ (left) and $\tilde{\lambda}_{i}$ (right) for $\epsilon=0.001$.

\section{NUMERICAL RESULTS}

\subsection{Experiment 1}

We examined the numerical method presented here on a benchmark problem posed on the unit square, subject to the nontrivial boundary conditions as depicted in Fig. 5. The basic mesh was made up of $20 \times 20$ rectangles, whose edges were parallel to the coordinate axes. The only exception is in Fig. 6 (right), in which we used $20 \times 10$ rectangular elements. We tested the method for high Peclet numbers (that is, $\epsilon=10^{-6}$ ) and three different convection fields: $\beta=(1,2), \beta=(1,1)$, and $\beta=(2,1)$. Since the basis functions in both the multiscale and the PMS method are comparable (see Figs. 2-4), they produce almost the same results. Therefore, we only display the numerical results obtained by the PMS method due to its little cost. 




FIG. 5: Problem description on square domain


FIG. 6: PMS approximations on nonaligned (left) and aligned (right) uniform rectangular mesh with $\beta=(1,2)$.

\subsubsection{Case 1. $f=0$}

In nonaligned meshes, we observed that the PMS method produced accurate results at the outflow boundaries, yet it did not capture well internal layers [Fig. 6 (left)]; in which case, the numerical solution presents oscillations in some parts of the domain close to the internal layer. Contrary to the PMS method, the PRFB method captures internal layers well; however, it displays some unphysical oscillations around the outflow (Brezzi et al., 1998b; Neslitürk, 2010). Motivated by this observation, the mix method RFB-MS, which has been proposed by Franca et al. (2005b), is based on the idea that the multiscale functions are used in the elements connected to the outflow boundaries and the RFB functions are used in the rest of the domain. We then applied the same strategy, yet, the approximate counterparts of the multiscale and RFB functions are used. The resulting numerical method is denoted by PRFB-PMS. In order to display the performance of our method, we compare it with the PRFB method on the uniform mesh in Figs. 7-9 and nonuniform mesh in Fig. 10. It is obvious that the proposed algorithms improve over the PRFB method. 



FIG. 7: PRFB (left) and PRFB-PMS (right) approximations with $\beta=(1,2)$.


FIG. 8: PRFB (left) and PRFB-PMS (right) approximations with $\beta=(1,1)$.

\subsubsection{Case 2. $f=1$}

In this part, we report some results for convection diffusion problem with nonzero source term. In order to approximate the enriching function $\phi_{f}$ in Eq. (16), we decompose $\phi_{f}=\phi_{f}^{1}+\phi_{f}^{2}$ such that

$$
\mathfrak{L} \phi_{f}^{1}=f \text { in } K, \quad \phi_{f}^{1}=0 \text { on } \partial K
$$





FIG. 9: PRFB (left) and PRFB-PMS (right) approximations with $\beta=(2,1)$.


FIG. 10: PRFB (left) and PRFB-PMS (right) approximations on nonuniform mesh with $\beta=(2,1)$.

and

$$
\mathfrak{L} \phi_{f}^{2}=0 \text { in } K, \quad \phi_{f}^{2}=v_{f} \text { on } \partial K
$$

Since $\phi_{f}^{1}$ vanishes on the element boundary, the PRFB method on the rectangular elements can be applied to Eq. (32) (Neslitürk, 2010). On the other hand, we skip the contribution of $\phi_{f}^{2}$ from algorithm (18) as our computational experiments show that its effect is negligible. Thus, the resulting modified scheme reads: Find $u_{i}$ such that 


$$
\sum_{i \in I_{0}} a\left(\lambda_{i}, \psi_{j}\right) u_{i}=\left(f, \psi_{j}\right)-a\left(\phi_{f}^{1}, \psi_{j}\right) \text { for all } j \in I_{0}
$$

We remark that this modified method is also a consequence of choosing the boundary condition as

$$
\mathcal{L}_{\partial K} u^{e}=-\mathcal{L}_{\partial K} u^{1} \text { on } \partial K, \quad u^{e}=0 \text { at the vertices of } K
$$

instead of Eq. (13). Thus, we employ the approximation of $\phi_{f}^{1}$ instead of $\phi_{f}$ in the mixed PRFB-PMS algorithm. In Figs. 11 and 12 we again compare the PRFB and PRFB-PMS methods for $f=1$ and the convection fields $\beta=(1,1)$ and $\beta=(2,1)$. As predicted, the PRFB-PMS method performs better than the PRFB in each case.


FIG. 11: PRFB (left) and PRFB-PMS (right) approximations with $\beta=(1,1)$ and $f=1$.
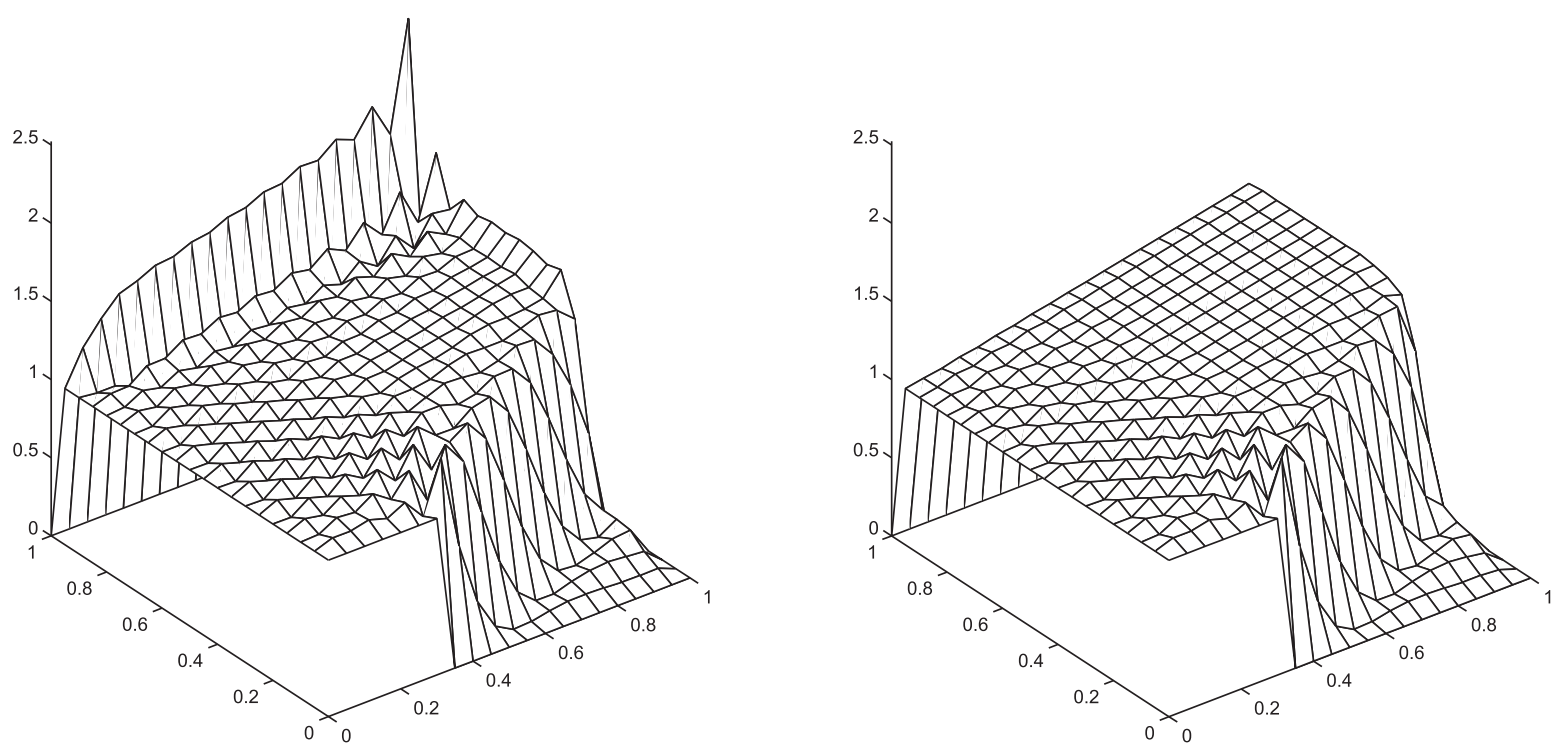

FIG. 12: PRFB (left) and PRFB-PMS (right) approximations with $\beta=(2,1)$ and $f=1$. 


\subsection{Experiment 2}

We then tested the proposed algorithm for a nonconstant flow field on an L-shaped domain with the boundary conditions depicted in Fig. 13(left). We take $\beta=(y, 1-x), \epsilon=0.005$ and $f=0$. The exact solution of the problem exhibited boundary layers near the outflow boundaries that are the upper side, right-upper side and below the corner of the domain Brezzi et al. (1998b). We discretized the domain into 300 uniform rectangular elements [Fig. 13(right)]. In computations, we used the average value of the flow field over the whole element; that is

$$
\left.\bar{\beta}_{i}\right|_{K}=\frac{1}{|K|} \iint_{K} \beta_{i} \mathrm{~d} x \mathrm{~d} y \text { for } i=1,2 .
$$

We applied the same approach used in Experiment 1 and observed that PRFB-PMS method was still able to produce better approximations than the PRFB for more complicated problem configurations (Figs. 14 and 15).


FIG. 13: Problem description on L-shape domain and the mesh employed.


FIG. 14: PRFB (left) and PRFB-PMS (right) approximations on L-shape domain. 



FIG. 15: Contour-lines of PRFB (left) and PRFB-PMS (right) approximations on L-shape domain.

\section{REFERENCES}

Brezzi, F. and Douglas, J., Stabilized mixed methods for the Stokes problem, Numer. Math., vol. 53, no. 1-2, pp. 225-235, 1988.

Brezzi, F., Franca, L. P., and Russo, A., Further considerations on residual-free bubbles for advective-diffusive equations, Comput. Methods Appl. Mech. Eng., vol. 166, pp. 25-33, 1998a.

Brezzi, F., Hauke, G., Marini, L. D., and Sangalli, G., Link-cutting bubbles for the stabilization of convection-diffusion-reaction problems, Math. Models Meth. Appl. Sci., vol. 13, pp. 3445-3461, 2003.

Brezzi, F., Hughes, T. J. D., Marini, L. D., Russo, A., and Süli, E., A priori error analysis of residual-free bubbles for advectiondiffusion problems, SIAM J. Numer. Anal., vol. 36, pp. 1933-1948, 1999.

Brezzi, F., Marini, D., and Russo, A., Applications of the pseudo residual-free bubbles to the stabilization of convection-diffusion problems, Comput. Methods Appl. Mech. Eng., vol. 166, pp. 51-63, $1998 \mathrm{~b}$.

Brezzi, F., Marini, D., and Russo, A., On the choice of a stabilizing subgrid for convection-diffusion problems, Comput. Methods Appl. Mech. Eng., vol. 194, pp. 127-148, 2005.

Brezzi, F. and Russo, A., Choosing bubbles for advection-diffusion problems, Math. Models Methods Appl. Sci., vol. 4, pp. 571587, 1994.

Brooks, A. N. and Hughes, T. J. R., Streamline upwind/Petrov-Galerkin formulations for convection dominated flows with particular emphasis on the incompressible Navier-Stokes equations, Comput. Methods Appl. Mech. Eng., vol. 32, pp. 199-259, 1982.

Franca, L. P. and Frey, S. L., Stabilized finite element methods: II. The incompressible Navier-Stokes equations, Comput. Methods Appl. Mech. Eng., vol. 99, no. 2-3, pp. 209-233, 1992.

Franca, L. P., Frey, S. L., and Hughes, T. J. R., Stabilized finite element methods: I. Application to the advective-diffusion model, Comput. Methods Appl. Mech. Eng., vol. 95, pp. 253-276, 1992.

Franca, L. P., Neslitürk, A. I., and Stynes, M., On the stability of residual-free bubbles for convection-diffusion problems and their approximation by two-level finite element method, Comput. Methods Appl. Mech. Eng., vol. 166, pp. 35-49, 1998.

Franca, L. P., Madureira, A. L., Tobiska, L., and Valentin, F., Convergence analysis of a multiscale finite element method for singularly perturbed problems, Int. Comput. Eng., vol. 3, no. 3, pp. 297-312, 2005a.

Franca, L. P., Madureira, A. L., and Valentin, F., Towards multiscale functions: Enriching finite element spaces with local but not bubble-like functions, Comp. Methods Appl. Mech. Eng., vol. 194, pp. 3006-3021, 2005b. 
Franca, L. P., Ramalho, J. V. A., and Valentin, F., Multiscale and residual-free bubble functions for reaction-advection-diffusion problems, Int. Comput. Eng., vol. 3, no. 3, pp. 297-312, 2005c.

Franca, L. P. and Valentin, F., On an improved unusual stabilized finite element method for the advective-reactive-diffusive equation, Comput. Methods Appl. Mech. Eng., vol. 190, pp. 1785-1800, 2000.

Harari, I. and Hughes, T. J. R., Stabilized finite element methods for steady advection-diffusion with production, Comput. Methods Appl. Mech. Eng., vol. 115, pp. 165-191, 1994.

Hughes, T. J. R., Franca, L. P., and Balestra, M., A new finite element formulation for computational fluid dynamics. 5. Circumventing the Babuska-Brezzi condition-A stable Petrov-Galerkin formulation of the Stokes problem accommodating equal-order interpolations, Comput. Methods Appl. Mech. Eng., vol. 59, no. 1, pp. 85-99, 1986.

Neslitürk, A. I., A stabilizing subgrid for convection-diffusion problem, Math. Models Meth. Appl. Sci., vol. 16, no. 2, pp. 211-231, 2006.

Neslitürk, A. I., On the choice of stabilizing subgrid for convection-diffusion problem on rectangular grids, Comput. Math. Appl., vol. 59, pp. 3687-3699, 2010

Sangalli, G., Global and local error analysis for the residual-free bubbles method applied to advection-dominated problems, SIAM J. Numer. Anal., vol. 38, pp. 1496-1522, 2000.

Volume 11, Number 4, 2013 